\title{
ORIGINAL
}

\section{CUIDADOS DE ENFERMERÍA EN HOSPITALIZACIÓN A DOMICILIO Y HOSPITALIZACIÓN CONVENCIONAL}

\author{
David Oterino de la Fuente (1), Salvador Peiró Moreno (1,2), Catalina Marchan Rodríguez (3) y \\ Manuel Ridao López (1) \\ (1) Instituto de Investigación en Servicios de Salud (IISS). \\ (2) Institut Valencià d'Estudis en Salut Pública (IVESP). \\ (3) Hospital Clínico-Universitario de Valencia.
}

\section{RESUMEN}

Fundamento: Los cuidados de enfermería tienen especial importancia en la hospitalización domiciliaria (HD), tanto por las características de los pacientes como por su transferencia hacia los cuidadores informales del paciente. El objetivo de este trabajo es analizar la cantidad y tipo de cuidados de enfermería administrados y requeridos por los pacientes en HD y hospitalización convencional (HC).

Metodo: Se utilizó el Project Research Nursing (PRN) para cuantificar el volumen y tipo de cuidados de enfermería administrados y requeridos en 2 grupos de pacientes (HD: 148 pacientes, 1776 estancias; HC: 148 pacientes, 1113 estancias) con características similares tratados en cada una de las alternativas a evaluación, analizándose las diferencias según modalidades de atención y características de los pacientes.

Resultados: Respecto a los cuidados administrados, los pacientes en HD (647,8 minutos/episodio) recibieron un menor volumen de cuidados que en $\mathrm{HC}$ (1030 minutos/episodio). Esta diferencia se debió fundamentalmente a que les fueron administrados menos cuidados básicos (HD: 96,6 min./episodio; HC: 464,3 in/episodio) y diagnósticos (HD: 84,4 in/episodio; HC: 167,3$)$. Por el contrario, los pacientes en HD recibieron una mayor cantidad de cuidados de comunicación (238,8 minutos, frente a 107,4 en HC). No se hallaron diferencias significativas entre cuidados administrados y requeridos.

Conclusiones: Los pacientes en HD reciben menos cuidados de enfermería que en $\mathrm{HC}$, fundamentalmente a expensas de los cuidados básicos (transferidos a los cuidadores) y de los cuidados asociados a la realización de pruebas diagnósticas (dependientes de los estilos de práctica médicos), aunque reciben mas cuidados de comunicación (educación sanitaria). La ausencia de diferencias entre los cuidados realmente administrados y los requeridos sugiere una adecuada calidad asistencial en ambas modalidades de hospitalización.

Palabras clave: Hospitalización a domicilio. Cuidados de enfermería.

Correspondencia

David Oterino de la Fuente

Instituto de Investigación en Servicios de Salud

El Puerto, 46

Santa María del Mar

Asturias

\section{ABSTRACT}

\section{Nursing Care in Home Hospitalisation and Conventional Hospitalization.}

Background: nursing care is of especial importance in hospital care in the home $(\mathrm{HH})$. This is as due to the characteristics of the patient as it is to the transfer to the informal carers for the patient. The aim of this study is to analyse the quantity and type of nursing care administered and required by patients in $\mathrm{HH}$ and conventional hospitalisation $(\mathrm{CH})$.

Methods: Project Research Nursing (PRN) was used to quantify the amount and types of nursing care administered and required in 2 groups of patients (HH: 148 patients, 1776 stays; $\mathrm{CH}$ : 148 paticnts, 1113 stays) having similar characteristics and treated in each way, together with how they evolved, analysing differences in terms of forms of attention and the characteristics of the patients.

Results: in terms of the care administered, those patients in HH (647.8 minutes / episode) received less care than those in $\mathrm{CH}$ (1030 minutes / episode). This difference was fundamentally due to the fact that they received less basic care (HH: 96.6 min./episode; $\mathrm{CH}: 464.3 \mathrm{~min} . / \mathrm{episode}$ ) and diagnostic work (HH: 84.4 min./episode; $\mathrm{CH}: 177.3$ ). On the other hand, patients in $\mathrm{HH}$ received a greater quantity of communicational care (238.8 min., as opposed to 107.4 in CH). No significant differences were found between the care administered and that which was required.

Conclusions: patients in $\mathrm{HH}$ received less nursing care than did those in $\mathrm{CH}$, mainly due to the fact that they received less basic care from nurses, as this work was transferred to their careers. They also received less care associated with diagnostic tests (depending on styles of medical practice), although they received more care in the form of communication (health education). The lack of differences between the care that was actually administered and that which was required suggests that the quality of care provided is sufficient in both forms of hospitalisation.

Key words: Home hospitalisation. Nursing care. 


\section{INTRODUCCIÓN}

Los cuidados de enfermería tienen especial importancia en la hospitalización domiciliaria (HD), tanto por las características de buena parte de los pacientes que utilizan esta modalidad de atención (pacientes ancianos con pluripatología, en la fase final del episodio de hospitalización) como porque parte de estos cuidados son transferidos a los cuidadores del paciente. Sin embargo, el volumen y tipo de cuidados de enfermería que se presta por las unidades de hospitalización a domicilio (UHD) es un tema poco estudiado.

El nivel de cuidados de enfermería requerido por un paciente puede estimarse por métodos directos e indirectos. Los métodos indirectos, como Medicus o Etxaquet ${ }^{1-3}$, están basados en un listado de indicadores seleccionados por expertos, que se consideran buenos predictores de necesidad de cuidados y permiten clasificar a los pacientes en diferentes categorías. La principal limitación de los métodos indirectos es su falta de validez externa que impide la comparación entre distintos hospitales o incluso del mismo hospital en distintos períodos de tiempo ${ }^{4}$. Los métodos directos, como el Project Research Nursing (PRN) ${ }^{5.6}$ o el Grace Reynolds Application and Study of $P_{E T O}{ }^{7}$, se basan en una lista de todos los cuidados de enfermería posibles, exhaustiva y mútuamente excluyente con el fin de no contabilizar doblemente los cuidados. El tiempo requerido para ejecutar las actividades que requiere cada cuidado se determina por consenso de expertos (tiempo normativo) o por cronómetro (tiempo utilizado). Los métodos directos tienen la ventaja de permitir comparar la cantidad y tipo de cuidados entre unidades de hospitalización o entre hospitales, siempre que la lista de cuidados contenga todas las acciones posibles. En este trabajo se ha utilizado un método directo, el PRN, ya que es uno de los mas utilizados en España y permite la comparación ulterior de los resultados con otros trabajos que pudieran realizarse.
Estos métodos se han utilizado fundamentalmente para la redistribución del personal de enfermería en las distintas unidades de hospitalización, bajo los supuestos de que ayudan a un reparto más equitativo de las cargas de trabajo, que aumenta la satisfacción del personal por mejora la calidad asistencial y la eficiencia en la atención ${ }^{8.9}$. Adicionalmente, conocer el volumen y tipo de cuidados requeridos por un grupo de pacientes permite su comparación con los cuidados realmente administrados, aproximándose a una medición de la calidad asistencial a través de la diferencia entre el nivel de cuidados requeridos según los estándares del hospital y los cuidados administrados ${ }^{8}$. Del mismo modo, puede conocerse la cantidad y tipo de cuidados transferidos a los cuidadores informales del paciente. El objetivo de este trabajo, que forma parte de un estudio mas amplio para evaluar la eficiencia de la HD frente a la hospitalización convencional $(\mathrm{HC})^{10}$, es cuantificar la cantidad y tipo de cuidados de enfermería en HD y $\mathrm{HC}$, analizando sus diferencias.

\section{SUJETOS Y MÉTODO}

Población y ámbito del estudio: Se midieron los cuidados de enfermería, requeridos y administrados, en 148 ingresos consecutivos (1776 estancias) de una Unidad de Hospitalización Domiciliaria (UHD) dependiente de un hospital de tercer nivel del Servicio Valenciano de Salud, y un grupo control integrado por otros 148 ingresos (1113 estancias) de características similares, hospitalizados en los mismos servicios de donde procedían los ingresados en el domicilio.

Cuidados de enfermería: son todas las actividades (administrativas y sobre el paciente) realizadas por el personal de enfermería para satisfacer las necesidades del paciente ingresado, los cuidados requeridos vienen determinados por las características clínicas y sociales de los pacientes y el estándar de calidad del hospital «que cuidados 
y como deben darse» y los cuidados dados son los que se dispensan en realidad «como se dan» ${ }^{4}$.

Criterios de inclusión: Para el grupo de pacientes en HD se exigió que provinieran del propio hospital, excluyéndose los pacientes que provenían directamente de atención primaria, consultas externas o urgencias. Para incluir un paciente como control se exigió pertenecer al mismo grupo patológico, de edad y sexo que el correspondiente caso, cumplir los criterios clínicos para ser admitido en la UHD especificados en los Protocolos de inclusión en hospitalización a domicilio en el Servicio Valenciano de Salud ${ }^{11}$, y llevar ingresado entre 7 y 14 días en el hospital. Estc último criterio se estableció al considerar que el episodio completo de hospitalización del paciente en HD contiene una parte en el hospital (estancia preUHD) que, por realizarse al inicio de la hospitalización, incluye las fases de mayor intensidad diagnóstica y terapéutica y, por tanto, de mayor intensidad en cuidados de enfermería. Para limitar esta dificultad los cuidados de enfermería de los pacientes ingresados, se evaluaron desde que fueron incluidos como controles en el estudio cuando ya llevaban ingresados un período similar (al menos 6 días) al que llevaban ingresados los pacientes al pasar a HD.

Todos los pacientes se seleccionaron prospectivamente hasta alcanzar el tamaño muestral requerido para el objetivo central del proyecto ${ }^{10}$ que, a los efectos del análisis actual, permitiría detectar diferencias superiores al $20 \%$ en tiempo de cuidados de enfermería, con un error tipo I del $5 \%$ y un error tipo II del $10 \%$.

Descripción de alternativas: a) la HD requiere que el personal médico y de enfermería se desplace al domicilio del paciente para realizar las consultas y prestar los cuidados que éste precise, quedando a cargo de los familiares los cuidados generales del pa- ciente, la higiene y la alimentación; incluye facilitar los fármacos y material sanitario, así como la realización de pruebas diagnósticas con traslado de las muestras o del paciente al hospital. b) la $\mathrm{HC}$ implica la permanencia del paciente en una cama del hospital e incluye todos los cuidados y tratamientos de enfermería, consultas médicas realizadas, fármacos, material sanitario y pruebas diagnósticas, así como la prestación de los servicios de soporte general correspondientes.

Instrumento de medida: Los cuidados de enfermería fueron medidos mediante el PRN, un instrumento diseñado en la Universidad de Montreal por el Equipe de Recherche Operationelle en Santé (EROS) en 1980 , revisado en $1987^{6,12}$, y actualmente utilizado en Canadá, Francia, Bélgica, Suiza y España ${ }^{4,13,14}$. En España ha sido validado en $1990^{\circ}$ y hasta la fecha ha sido utilizado en la evaluación de las cargas de trabajo de enfermería en más de 100 unidades de hospitalización de diferentes hospitales (Carrillo E, comunicación personal), si bien la mayor parte de los resultados no han sido publicados por formar parte de actividades de consultoría.

El PRN contiene un listado exhaustivo y mutuamente excluyente de cuidados de enfermería posibles. A partir de 99 actos específicos estima el tiempo atribuido a cada actividad según el nivel de ayuda requerido, el número de veces que ha de ser realizada y el número de personas necesario para su realización, hasta totalizar 249 actividades distintas $^{12}$. El PRN permite medir la cantidad de los cuidados requeridos y los cuidados dados a los pacientes - en tiempo-y también el tipo de cuidados que se requieren o se dan. Clasifica a los pacientes en 15 niveles según la cantidad de cuidados y discrimina entre cuidados básicos (alimentación, higiene, movilización, respiración, eliminación), cuidados técnicos (diagnostico y tratamiento) y de comunicación (educación para la salud, información al paciente). La medición es realizada habitualmente por personal de enfermería previamente adiestrado en el uso 
del instrumento a través de la información que consta en la historia clínica, y la revisión puede hacerse de forma retrospectiva, prospectiva o concurrente.

Variables: Del paciente: edad; sexo; estado civil; nivel de estudios (superior, medio, primarios, sin estudios); situación laboral (trabaja, no trabaja, pensionista); disponibilidad de ayuda por trabajadores sociales (si/no); y presencia de pluripatología (existencia de uno o más de un cuadro patológico al ingreso).

Del episodio de hospitalización: tipo de ingreso en el hospital (urgente o programado); grupo patológico (clasificación en 4 grupos utilizada por la UHD: agudo, crónico, quirúrgico, oncológico); servicio hospitalario (en el caso de la UHD, servicio del que proviene el paciente); destino al alta (domicilio, consultas externas, hospital de crónicos, reingreso, éxitus); duración de la estancia (del episodio completo y desde la inclusión de en HD o en el estudio); adecuación del ingreso según el protocolo de evaluación del uso inapropiado de la hospitalización (apropiado o inapropiado).

Proceso de recogida de datos: $\mathrm{El}$ estudio fue aprobado por la Comisión de Investigación del Hospital y contó con la colaboración de la Dirección del centro y las unidades participantes. Los datos referidos a las características del paciente y del episodio de hospitalización de los enfermos ingresados en HD fueron recogidos por el personal de enfermería de la UHD, mientras que en el grupo control los datos los recogió una enfermera participante en el estudio. La selección de los controles fue realizada por el personal de enfermería de la UHD que evalúa habitualmente las peticiones de traslado de enfermos hospitalizado a la HD. Los cuidados de enfermería requeridos y administrados a todos los pacientes incluidos en el estudio, tanto en HD como en $\mathrm{HC}$, fueron recogidos por una enfermera con experien- cia en el uso del PRN revisando la información disponible en la historia clínica.

Análisis: Se ha realizado un análisis descriptivo de las características de los pacientes, episodio de hospitalización, actividades asistenciales. Para el análisis bivariante se emplearon las pruebas de $\chi^{2}$ y $t$ de Student, según se tratara de variable categóricas o continuas. En este último caso, y dado que la mayoría de las variables no cumplían los supuestos de distribución normal y homogeneidad de varianzas entre grupos, se realizó también la prueba no paramétrica de MannWhitney para muestras independientes (pese a que en el diseño del trabajo existe un alto grado de apareamiento, se optó por utilizar las -usualmente mas conservadoraspruebas estadísticas para discños no apareados.

\section{RESULTADOS}

Los pacientes en HD y $\mathrm{HC}$ no presentaron diferencias estadísticamente significativas en cuanto a las características personales y de los episodios de hospitalización estudiados a excepción del destino al alta y la estancia media (Tabla 1). El destino al alta mas frecuente fue hacia atención primaria o consultas externas en ambos grupos (HD. $85,7 \%$, HC: $73,3 \%$ ), pero en HC las altas voluntarias, los reingresos y el traslado a hospitales de crónicos fueron mas numerosos. La estancia media (EM) del episodio completo de hospitalización, incluyendo en su caso las estancias en el hospital de los pacientes que posteriormente pasaron a la UHD, fue de 29,7 días (Intervalo de confianza del $95 \%$, IC95\%: 26,93-32,42) para los pacientes en HD y 23,0 días (IC95\%: 20,57$25,47)$ para los pacientes en HC. La EM en HD fue de 12,0 días (IC95\%: 10,96-13,04), por 7,5 días (IC95\%: 6,33-8,71) de EM posterior al reclutamiento de los controles en HC. La estancia total y en UHD mostró diferencias significativas entre ambos grupos, pero no así la estancia pre-UHD, utilizada para la selección de controles. 
Tabla 1

Características de los pacientes y episodios de hospitalización

\begin{tabular}{|c|c|c|c|}
\hline & & Domicilio (\%) & Hospital (\%) \\
\hline Edad* & $\begin{array}{l}15-44 \text { años } \\
45-64 \text { años } \\
65-79 \text { años } \\
\geq 80 \text { años }\end{array}$ & $\begin{array}{l}14(9,5) \\
38(25,7) \\
66(44,6) \\
30(20,3)\end{array}$ & $\begin{array}{l}14 \quad(9,5) \\
38(25,7) \\
66(44,6) \\
30(20,3)\end{array}$ \\
\hline Estado civil & $\begin{array}{l}\text { Casado } \\
\text { Soltero } \\
\text { Separado/Divorciado } \\
\text { Viudo }\end{array}$ & $\begin{array}{rr}90(61,2) \\
14(9,5) \\
5(3,4) \\
38(25,9)\end{array}$ & $\begin{array}{rr}86 & (58,1) \\
13 & (8,8) \\
4 & (2,7) \\
45 & (30,4)\end{array}$ \\
\hline Nivel de estudios & $\begin{array}{l}\text { Superiores } \\
\text { Medios } \\
\text { Primarios/Sin estudios }\end{array}$ & $\begin{array}{r}8(5,4) \\
21(14,3) \\
118(80,3)\end{array}$ & $\begin{array}{r}2(1,4) \\
15(10,1) \\
131(88,5)\end{array}$ \\
\hline Situación laboral & $\begin{array}{l}\text { Trabaja } \\
\text { No Trabaja } \\
\text { Pensionista }\end{array}$ & $\begin{array}{l}20(13,6) \\
29(19,7) \\
98(66,7)\end{array}$ & $\begin{array}{l}20(13,6) \\
39(26,5) \\
88(59.9)\end{array}$ \\
\hline Sexo* & $\begin{array}{l}\text { Hombre } \\
\text { Mujer }\end{array}$ & $\begin{array}{l}79(53,4) \\
69(46,6)\end{array}$ & $\begin{array}{l}79(53,4) \\
69(46,6)\end{array}$ \\
\hline Ayuda social & $\begin{array}{l}\text { Sí } \\
\text { No }\end{array}$ & $\begin{array}{r}5(3,5) \\
136(96,5)\end{array}$ & $\begin{array}{r}7(5,4) \\
122(94,6)\end{array}$ \\
\hline Pluripatología & $\begin{array}{l}\text { Sí } \\
\text { No }\end{array}$ & $\begin{array}{l}58(45,0) \\
71(55,0)\end{array}$ & $\begin{array}{l}76(54,7) \\
63(45,3)\end{array}$ \\
\hline Grupo patológico & $\begin{array}{l}\text { Quirúrgico } \\
\text { Crónico } \\
\text { Agudo } \\
\text { Oncológico }\end{array}$ & $\begin{array}{l}83(56,1) \\
41(27,7) \\
12(8,1) \\
12 \quad(8,1)\end{array}$ & $\begin{array}{l}83(56,1) \\
41(27,7) \\
12 \quad(8,1) \\
12 \quad(8,1)\end{array}$ \\
\hline Tipo ingreso en el hospital & $\begin{array}{l}\text { Urgente } \\
\text { Programado }\end{array}$ & $\begin{array}{l}99(73,6) \\
36(26,7)\end{array}$ & $\begin{array}{r}113(77,9) \\
32(22,1)\end{array}$ \\
\hline Destino al alta** & $\begin{array}{l}\text { Atención Primaria } \\
\text { Reingreso Hospital } \\
\text { Hospital Crónicos } \\
\text { Alta Voluntaria } \\
\text { F́xitus }\end{array}$ & $\begin{array}{rr}126 & (85,7) \\
8 & (5,4) \\
4 & (2,7) \\
0 & (0,0) \\
9 & (6,1)\end{array}$ & 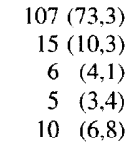 \\
\hline Servicio hospitalario* & $\begin{array}{l}\text { Quirúrgicos } \\
\text { Médicos }\end{array}$ & $\begin{array}{l}84(56,8) \\
64(43,2)\end{array}$ & $\begin{array}{l}84(56,8) \\
64(43,2)\end{array}$ \\
\hline $\begin{array}{l}\text { Duración de la estancia en UHD o pe- } \\
\text { ríodo equivalente en el hospital** }\end{array}$ & $\begin{array}{l}\text { Menos de } 7 \text { días } \\
\text { de } 7 \text { a } 14 \text { días } \\
\text { de } 14 \text { a } 21 \text { días } \\
\text { Más de } 21 \text { días }\end{array}$ & $\begin{array}{l}29(19,6) \\
79(53,4) \\
28(18,9) \\
12 \quad(8,1)\end{array}$ & 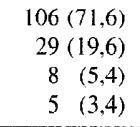 \\
\hline Número pruebas diagnósticas ** & $\begin{array}{l}\text { Ninguna prueba } \\
\text { De } 1 \text { a } 3 \text { pruebas } \\
\text { Más de } 3 \text { pruebas }\end{array}$ & $\begin{array}{r}117(79,1) \\
17(11,5) \\
14(9,5)\end{array}$ & $\begin{array}{l}49(33,1) \\
49(33,1) \\
50(33,8)\end{array}$ \\
\hline Número consultas médicas ** & $\begin{array}{l}\text { Menos de } 2 \text { consultas } \\
\text { De } 3 \text { a } 6 \text { consultas } \\
\text { Más de } 6 \text { consultas }\end{array}$ & $\begin{array}{r}79(53,4) \\
60(40,5) \\
9 \quad(6,1)\end{array}$ & $\begin{array}{l}19(12,8) \\
79(53,4) \\
50(33,8)\end{array}$ \\
\hline
\end{tabular}

* Variable utilizada como criterio de selección de controles; ** $\mathrm{p}<0,05$.

Los médicos realizaron menos consultas pero de mayor duración en el domicilio que en el hospital (HD: 3,1 visitas/episo- dio de 23,6 minutos; HC: 7,3 visitas/episodio de 9,4 minutos). El número de visitas que el personal de enfermería realiza en el 
hospital no fue contabilizado al considerarse que eran muy numerosas a lo largo del día, no siempre se registran en la historia clínica y la medida de interés era el tiempo global dedicado al paciente. En el domicilio el personal de enfermería realizó 7,9 visitas/episodio.

La cantidad total de cuidados de enfermería administrados por episodio de hospitalización (tabla 2), sin contabilizar el tiempo de transporte, fue significativamente inferior en el domicilio que en el hospital (HD: 647,8 min.; IC95\%: 487,8807,9; HC: 1030,0 min.; IC95\%: 857,81202,2). En el domicilio el volumen de cuidados básicos fue significativamente menor que en el hospital, salvo en lo referente a cuidados de climinación; por el contrario el tiempo empleado en comunicación fue superior en $\mathrm{HD}$ que en $\mathrm{HC}$ $(\mathrm{HD}=238,8 ; \mathrm{HC}=107,4)$. Para los cuidados técnicos, en conjunto, no se observaron diferencias significativas, si bien dentro de ellos los cuidados relacionados con el diagnóstico fueron significativamente inferiores en $\mathrm{HD},(\mathrm{HD}=84,4 ; \mathrm{HC}=167,3)$.
Los cuidados requeridos mostraron un comportamiento similar, con menores necesidades en HD, a expensas de los cuidados básicos, y mayores necesidades de comunicación. No se observaron diferencias significativas entre los cuidados de enfermería que requerían los pacientes y los cuidados recibidos, tanto en HD como en HC.

El volumen total de cuidados administrados y requeridos, tanto en el domicilio como en el hospital (tabla 3), no mostró diferencias según la edad, sexo, estado civil, nivel de estudios, situación laboral, ayuda social, pluripatología, tipo de ingreso, servicio hospitalario y necesidad del ingreso. En el domicilio los pacientes pertenecientes al grupo de oncología y los que se remitieron a un hospital de crónicos recibieron, significativamente $(\mathrm{p}<0,05)$, más cuidados que el resto. En el hospital los pacientes que finalmente fallecieron y los pacientes a los que se les realizaron más de tres pruebas diagnósticas fueron quienes más cuidados recibieron. Tanto en el domicilio como en el hospital la cantidad de cuidados dados y requeridos se asoció significativamente a la mayor duración

Tabla 2

Tipo y tiempo (minutos) de cuidados de enfermería por episodio de hospitalización

\begin{tabular}{|c|c|c|c|c|c|c|c|c|}
\hline & \multicolumn{4}{|c|}{ Cuidados requeridos } & \multicolumn{4}{|c|}{ cuidados administrados } \\
\hline & \multicolumn{2}{|c|}{ domicilio } & \multicolumn{2}{|c|}{ hospital } & \multicolumn{2}{|c|}{ domicilio } & \multicolumn{2}{|c|}{ hospital } \\
\hline & minutos & $1 \mathrm{C95 \%}$ & minutos & IC $95 \%$ & minutos & $I C_{95} \%$ & minutos & $I_{45 \%}$ \\
\hline Básicos* & 122,8 & $99,1-146,5$ & 487,8 & $382,2-593,4$ & 96,6 & $55,4-137,9$ & 464,3 & $354,1-574,4$ \\
\hline Alimentación* & 20,4 & $16,9-23,9$ & 111,7 & $90,7-132,6$ & 8,5 & $1,6-15,3$ & 95,0 & $78,7-111,4$ \\
\hline Eliminación & 50,4 & $29,2-71,7$ & 86 & $60,3-111,7$ & 45,9 & $21,6-70,3$ & 69,6 & $45,6-93,5$ \\
\hline Higiene** & 27,9 & $25,9-30,8$ & 187,6 & $157,8-217,4$ & 13,5 & $1,9-25,1$ & 170,6 & $140,5-200,7$ \\
\hline Movilización* & 7,6 & $5,1-10,2$ & 58,1 & $23,4-92,9$ & 13,5 & $3,1-23,9$ & 85,9 & $37,0-134,9$ \\
\hline Respiración* & 16,3 & 8,524 & 44,2 & $25,3-62,2$ & 15,3 & $6,9-23,7$ & 43.2 & $26,1-60,2$ \\
\hline Técnicos & 312,4 & $258,9-365,9$ & 430,1 & $358,6-501,6$ & 309,7 & $214,2-405,3$ & 458,4 & $381,0-535,7$ \\
\hline Diagnóstico* & 200,8 & $162-239,7$ & 273 & $216,3-329,8$ & 84,4 & $67,9-101,0$ & 167,3 & $145,6-189,1$ \\
\hline Tratamiento & 111,5 & $93-130,1$ & 157 & $134,5-179,2$ & 225,3 & $137,8-312,8$ & 291,1 & $227,9-354,2$ \\
\hline Comunicación $^{*}$ & 162,1 & $135,5-188,8$ & 107,7 & 92,9-122,5 & 238,8 & $186,7-290,9$ & 107,4 & $91,4-123,4$ \\
\hline TOTAL* & 597,5 & $509,1-685,8$ & $1.025,7$ & $856,5-1.194,9$ & 647,8 & $487,6-807,9$ & 1.030 & $857,8-202,2$ \\
\hline
\end{tabular}

*: $\mathrm{P}<0,05 ;$ IC95\%: Intervalo de confianza del $95 \%$ 
Tabla 3

Tiempo de cuidados de enfermería según características del paciente y del episodio de hospitalización

\begin{tabular}{|c|c|c|c|c|c|}
\hline & & \multicolumn{2}{|c|}{ Domicilio } & \multicolumn{2}{|c|}{ Hospital } \\
\hline & & Administrados & Requeridos & Administrados & Requeridos \\
\hline \multirow[t]{2}{*}{ Edad } & Menos de 65 años & 745,4 & 691,0 & 878,5 & 932,4 \\
\hline & Más de 65 años & 594,9 & 543,6 & $1.112,00$ & $1.076,8$ \\
\hline \multirow[t]{2}{*}{ Sexo } & Hombre & 596,0 & 550,0 & $1.061,40$ & $1.053,6$ \\
\hline & Mujer & 717,2 & 654,4 & 994,00 & 993,3 \\
\hline \multirow[t]{4}{*}{ Estado civil } & Casado & 694,8 & 601,2 & 119,90 & $1.043,1$ \\
\hline & Soltero & 331,4 & 387,5 & 421,30 & 790,0 \\
\hline & Separado/Viudo & 647,0 & 561,0 & 276,00 & 517,5 \\
\hline & Viudo & 708,9 & 699,9 & $1.100,80$ & $1.106,1$ \\
\hline \multirow[t]{3}{*}{ Nivel estudios } & Superiores & 784,4 & 510,0 & 822,50 & $1.345,0$ \\
\hline & Medios & 434,5 & 536,3 & 970,00 & 946,3 \\
\hline & Primarios & 690,6 & 620,5 & $1.040,00$ & $1.030,0$ \\
\hline \multirow[t]{3}{*}{ Situación laboral } & Trabaja & $1.048,7$ & 719,3 & 822,20 & 792,7 \\
\hline & No trabaja & 646,3 & 563,8 & 856.00 & 951,1 \\
\hline & Pensionista & 585,8 & 591,3 & $1.152,00$ & $1.109,0$ \\
\hline \multirow[t]{2}{*}{ Tipo ingreso } & Urgente & 582,9 & 577,3 & 1.083 .6 & $1.069,6$ \\
\hline & Programado & 896,3 & 720,8 & 808,4 & 802,3 \\
\hline \multirow[t]{2}{*}{ Necesidad del ingreso } & Apropiadas & 654,3 & 600,0 & $1.023,70$ & $1.028,0$ \\
\hline & Inapropiadas & 210,0 & 285,0 & $1.108,20$ & 997,7 \\
\hline \multirow[t]{2}{*}{ Ayuda social } & Sí & 449,0 & 901,0 & $1.182,80$ & 975,7 \\
\hline & No & 656,6 & 581.6 & 978.90 & 995.4 \\
\hline \multirow[t]{2}{*}{ Pluripatología } & Sí & 521,6 & 558,9 & $1.017,50$ & $1.000,1$ \\
\hline & No & 691,4 & 624,3 & $1.000,70$ & $1.018,9$ \\
\hline \multirow[t]{5}{*}{ Destino al alta* } & Atención Primaria & 623,8 & 591,5 & 784,9 & 792,3 \\
\hline & Reingreso Hospital & 523,6 & 538,6 & $1.532,6$ & $1.575,6$ \\
\hline & Hospital Crónicos & $2.090,0$ & $1.196,2$ & $1.418,8$ & $1.199,4$ \\
\hline & Alta Voluntaria & 0,0 & 0,0 & $1.623,0$ & $1.831,0$ \\
\hline & Éxitus & 415,0 & 463,7 & $2.358,5$ & $2.223,6$ \\
\hline \multirow[t]{4}{*}{ Grupo patológico* } & Quirúrgico & 642,6 & 578,3 & 926,2 & 930,8 \\
\hline & Crónico & 593,3 & 620,4 & 884,5 & $1.011,6$ \\
\hline & Agudo & 404,7 & 562,9 & $1.119,6$ & $1.094,5$ \\
\hline & Oncológico & $1.459,1$ & 790,5 & $1.570,4$ & $1.440,0$ \\
\hline \multirow[t]{2}{*}{ Servicio hospitalario } & Quirúrgicos & 696.9 & 587,0 & $1.056,5$ & $1.058,4$ \\
\hline & Médicos & 591,0 & 610,9 & 995,1 & 983,3 \\
\hline \multirow[t]{4}{*}{ Estancias en uhd* } & Menos de 7 días & 245,9 & 312.9 & 626,9 & 612,7 \\
\hline & Entre 7 y 14 días & 424,6 & 442,9 & $1.414,1$ & $1.386,8$ \\
\hline & Entre 14 y 21 días & $1.031,0$ & 922,0 & 3.195 .6 & $3.359,3$ \\
\hline & Más de 21 días & $1.841,0$ & $1.267,5$ & $3.881,0$ & $3.871,00$ \\
\hline \multirow[t]{3}{*}{ Número de consultas médicas* } & Hasta 2 consultas & 366,8 & 446,8 & 795,0 & 781,8 \\
\hline & De 3 a 6 consultas & 790,2 & 697,2 & 672,4 & 657,9 \\
\hline & Más de 6 consultas & $2.016,2$ & $1.136,2$ & $1.684,2$ & $1.692,2$ \\
\hline \multirow[t]{3}{*}{ Número pruebas analíticas* } & Ninguna prueba & 635,2 & 591,7 & 770,5 & 740,8 \\
\hline & De 1 a 3 pruebas & 777,8 & 585,3 & 992,9 & 930,2 \\
\hline & Más de 3 pruebas & 610.4 & 650,4 & 1.320 .6 & 1.392 .9 \\
\hline
\end{tabular}

$*$ : $p<0.05$. 
de la estancia, y al mayor número de consultas médicas.

\section{DISCUSIÓN}

La cantidad total de cuidados de enfermería administrados a los pacientes por episodio de hospitalización fue significativamente menor en el domicilio (647 minutos) que en el hospital (1030), resultado esperable ya que una parte importante de los cuidados que tiene asignados el personal de enfermería en $\mathrm{HC}$, son transferidos a los familiares o cuidadores en la HD. Dado que los cuidados básicos fueron siempre inferiores en $\mathrm{HD}$ que en $\mathrm{HC}$, es lógico suponer que es este tipo de cuidados el que se transfiere a los cuidadores. Por el contrario el tiempo empleado en comunicación fue significativamente superior en $\mathrm{HD}$, aspecto probablemente relacionado con la mayor importancia que se da a la educación en autocuidados (capacitar a la familia para administrar los cuidados, identificar signos y síntomas de riesgo, etc.) en esta modalidad de atención. La mayor cantidad de cuidados diagnósticos en HC podría estar relacionada con la mayor intensidad diagnóstica en el hospi$\operatorname{tal}^{12,15}$, mientras que las escasas diferencias en los cuidados de tratamiento orientarían hacia la homogeneidad de los 2 grupos de pacientes.

Respecto a los cuidados requeridos por los pacientes, las diferencias observadas entre la HD y la HC son muy similares a las observadas en los cuidados dados: menor necesidad total de cuidados en el domicilio, con menor necesidad de cuidados básicos y técnicos y mayor necesidad de comunicación. Hay que señalar que los cuidados de enfermeria medidos por el PRN están muy influenciados por la intensidad diagnóstica y terapéutica, bajo responsabilidad del médico y, por tanto, las diferencias en los estilos de práctica de los médicos se trasmitirán a los cuidados de enfermería necesarios y serán captadas por el PRN como diferencias entre los cuidados de enfermería (por ejemplo, si un médico ordena una determinada prueba diagnóstica, el PRN captará como cuidados necesarios todos los referidos a la preparación del paciente para realizar esta prueba: dieta, higiene, tratamiento, transporte, etc.).

Las diferencias entre HD y $\mathrm{HC}$ son explicables si las pautas de cuidados en el domicilio difieren del hospital, aspecto probable dadas las características de la HD donde el personal de enfermería se desplaza -habitualmente una vez al día- al domicilio del paciente, parte de los cuidados son transferidos a los cuidadores y se aumenta el tiempo empleado en comunicación (educación para la salud, que los cuidadores conozcan los cuidados requeridos por el paciente, como administrárselos y los signos de alarma de una posible complicación o descompensación del proceso). El hecho de que no se observen diferencias entre los cuidados requeridos y los cuidados dados, sugiere una buena calidad de los cuidados de enfermería en las dos alternativas de hospitalización ${ }^{8}$.

El mayor volumen de cuidados parece asociado a una mayor gravedad del paciente, sugerida por la mayor duración de la estancia, numero de consultas y pruebas diagnosticas, y coste de tratamiento. El hecho de que no se encontraran diferencias de cuidados en función de la edad ${ }^{13}$, esta probablemente relacionado con las características de los pacientes en HD (o susceptibles de HD), que difieren de la generalidad de los pacientes ingresados en un hospital dc agudos (su edad media es muy superior y los cuidados se miden al final de la fase evolutiva del proceso eliminando la parte aguda que se realiza bajo ingreso convencional). El mayor volumen de cuidados en los pacientes de HD que fueron trasladados a un hospital de crónicos podría estar en relación con una posible mayor gravedad de estos pacientes, pero también con la falta de cuidadores o poca colaboración por parte de estos. Por ultimo las diferencias observadas entre $\mathrm{HD}$ y $\mathrm{HC}$ en los 
cuidados de los pacientes que fallecieron podrían ser debidas a los diferentes estilos de práctica médica entre las dos modalidades de hospitalización.

Entre las limitaciones a considerar en este estudio hay que citar, en primer lugar, la asunción realizada en el diseño sobre la igualdad en calidad y efectividad del tratamiento médico y cuidados de enfermería entre ambas alternativas. Diversos estu$\operatorname{dios}^{16-20}$ han señalado algunas ventajas de la HD no consideradas en este trabajo, especialmente las relacionadas con la satisfacción y calidad de vida de los pacientes (menor interferencia en la vida diaria, mayor independencia, menor sensación de enfermedad, disminución de pérdida de horas de trabajo o escuela y, en pacientes geriátricos, mejora la situación funcional y los indicadores de salud general). Los estudios sobre calidad de la asistencia medida como disminución de infecciones, complicaciones, mortalidad y morbilidad (número e ingresos hospitalarios en el último año) ofrecen, por contra, resultados contradictorios $^{21}$.

La principal limitación del estudio reside, no obstante, en la comparabilidad de los 2 grupos de pacientes. A pesar de que las diferencias en características personales, sociales, y del episodio de hospitalización entre grupos son escasas, cabe la posibilidad de que los criterios de selección de controles no hayan conseguido obtener dos poblaciones suficientemente homogéneas respecto a su gravedad, que sería mayor para los pacientes hospitalizados y justificaría el mayor nivel de cuidados. Esta posibilidad, siempre presente cuando el diseño no es aleatorio, es muy improbable que pueda justificar la magnitud de las diferencias halladas, poco explicables incluso asumiendo diferencias entre los grupos mucho mayores que las esperables por la aplicación de los estrictos criterios de selección de controles y, en todo caso, debería mostrarse más en los cuidados técnicos que en los básicos.
El PRN presenta diversas limitaciones que es necesario tener en cuenta para interpretar los resultados obtenidos ${ }^{8,9}$. En su uso habitual, la medición de cargas de enfermería se realiza en unidades de hospitalización completas, durante las 24 horas del día con el fin de observar las diferencias entre los distintos turnos de trabajo, y habitualmente a lo largo de una semana para identificar las variaciones diarias. El PRN, por tanto, no está adaptado para seguir pacientes individuales durante todo el episodio de hospitalización e ingresados en distintas unidades de hospitalización, que mantienen protocolos distintos en como aplicar los cuidados, diferencias en la cantidad de personal de enfermería disponible - relación entre personal requerido y personal disponible- y en la calidad de éste, entendida como proporción de personal de enfermería titulado y de enfermería auxiliar. Igualmente, en su aplicación a la HD, el PRN mide los cuidados para las 24 horas, aunque que el personal de enfermería solo se desplace una vez al día al domicilio del enfermo para administrarle los cuidados. Esto implica que la asignación de tiempos atribuida por el PRN (en función del tipo de actividad y la frecuencia de la intervención) pueda diferir de la real, pero la descripción del tipo de cuidados y la relación entre ellos se puede considerar fiable.

Las principales aportaciones de este estudio radican en identificar y cuantificar las diferencias entre el nivel y tipo de cuidados de enfermería que se dan en HD y HC (lo que podría ser útil para la planificación de la cantidad de personal de enfermería necesario para administrar los cuidados y la formación del personal de enfermería adscrito a las UHD) y en mostrar una adecuada calidad de los cuidados de enfermería bajo esta modalidad. No obstante, sería conveniente la realización de más estudios que midieran el nivel de dependencia de los pacientes en HD y los cuidados de enfermería que requieren, una mayor definición de los cuidados que se administran y los que son transferidos a los 
cuidadores, y una mejor adaptación y validación del PRN para su uso en hospitalización domiciliaria.

\section{AGRADECIMIENTOS}

El personal medico y de enfermería de la Unidad de Hospitalización Domiciliaria del Ilospital Clínico Universitario de Valencia colaboró en todo momento en la recogida de datos. La firma Bossard SA facilitó el uso del Project Research Nursing y Esteban Carrillo y Mercé Estrems, nos prestaron una importante ayuda en el análisis e interpretación de algunos datos. La Dirección del Hospital Clínico Universitario de Valencia impulsó este estudio y facilitó los medios para desarrollarlo. $\mathrm{Ob}-$ viamente, ninguno de ellos, ni las Instituciones en que trabajan los autores, tiene responsabilidad sobre los posibles errores contenidos en el trabajo, ni comparten necesariamente sus contenidos.

\section{BIBLIOGRAFIA}

1. Jelineck RC. A new approach to the analysis of nursing activities. Hospitals JAHA 1966; 40: 89-91.

2. Jelineck RC. Estructural model for the patient care operation. Health Serv Res 1967; 2: 22642.

3. Exchaquet NF, Zublin L. Guide pour le calcul de la dotation en personal soignant des services des malades des hopitaux generaux. Berna: Etude des soins infirmiers en Suisse; 1975.

4. Tilquin C., Ferrús L., Portella E. Estrategias de medida de los cuidados de enfermería. Gac Sanit $1992 ; 6: 71$.

5. Tilquin C, Carle J, Saulinier D.. PRN-80. La mesure du niveau des soins infermiers requis. Montreal: EROS; 1980.

6. Tilquin C, Carle J, Saulinier D. PRN-87. La mesure du niveau des soins infermiers requis. Montreal: EROS; 1987.
7. Poland M, English N. PETO: A system for assessing and meetting patient care needs. Am J Nurs 1970; $70: 1479-82$.

8. Tilquin C, Roussel B, Laisney G, Ferrús L. Produire des indicateurs de charge temporelle de soins. Objetif Soins 1992; 3: 22-29.

9. Ferrús L, Portella E, Mata R. Determinación de estándares sobre cuidados enfermeros. Rev Rol Enfermería 1991; 153: 37-42.

10. Oterino de la Fuente D, Ridao M, Peiro S, Marchan C. Hospitalización a domicilio y hospitalización convencional. Una evaluación económica. Med Clin (Barc) 1997; 109: 207-211.

11. Servicio Valenciano de Salud. Protocolos de inclusión en hospitalización a domicilio en el Ser vicio Valenciano de Salud (mimeo). Documento HD-PI 1. Valencia: Servicio Valenciano de Salud: 1993.

12. Tilquin C, Saulnier D, Vanderstaeten G. El método PRN. Rev Rol Enfermería 1988; 119: 41-46.

13. Ferrus L, Tarruella M, Ruza, Penella J. Caracteristiques des soins infirmiers aux personnes agées dans des hopitaux de courte durée. Health systems. The challenge of change. Fifth International Conference on System Science in Health Care. Ginebra; 1995.

14. Samper M. Cargas de trabajo en unidades de enfermería: Un estudio practico. Rev Rol Enfermería 1986; 65-66: 56-63.

15. Oterino D, Peiró S, Ridao M, Marchan C. Variations in diagnostic and therapeutic intensity between home and conventional hospitalisation. Int J Qual Health Care 1997; en prensa.

16. Com-Ruelle, Raffy N. Les patients hospitalisés à domicile en 1.992. Paris: Centre de Recherche d'Etude et documentacion en economie de la Santé (CREDES); 1994.

17. Mediano C, Guillén M, Aranda E, Pérez F, Peiró $\mathrm{S}$. Como en casa en ningún sitio. Satisfacción de los pacientes hospitalizados a domicilio. $\mathrm{Rev} \mathrm{Ca}$ lidad Asistencial 1995; 1: 13-18.

18. Dummings JE, Hughes SL. Cost-effectiveness of Veterans Administration hospital-based home care. A randomized clinical trial. Arch Intern Med 1990; 150: 1274-80.

19. O'Cathain A. Evaluation of a hospital at home scheme for the early discharge of patients with fractured neck of femur. J Public Health Med 1994; 16: 205-10. 
20. Locutura J. Pertinencia de la estancia en un servicio de hospitalización a domicilio. Estudio retrospectivo de un año. I Jornada de Asistencia a Domicilio en el Area de Salud. Madrid: Ministerio de Sanidad y Consumo; 1988.
21. Deschamps DL, Tilquin C, Trudel R, Chevalier $S$, Poirier L. Un programmme public innovant: 1»hospitalisation à domicile. Montréal: Equipe de Recherche Opérationelle en Santé. Montréal: Université de Montréal; 1988. 